\title{
Health Informatics Learning Objectives on an Interoperable, Collaborative Platform
}

\author{
Cord SPRECKELSEN ${ }^{\mathrm{a}, 1}$, Ulrike SCHEMMANN ${ }^{\mathrm{b}}$, Lo An PHAN-VOGTMANNª, \\ André SCHERAG ${ }^{\mathrm{a}}$, Alfred WINTER ${ }^{\mathrm{c}}$ and Birgit SCHNEIDER ${ }^{\mathrm{c}}$ \\ ${ }^{a}$ Institute of Medical Statistics, Computer and Data Sciences, \\ Jena University Hospital, Germany \\ b Institute of Medical Informatics, RWTH Aachen, Germany \\ ' Institute for Medical Informatics, Statistics, and Epidemiology, \\ University of Leipzig, Germany
}

\begin{abstract}
Catalogues of learning objectives for Biomedical and Health Informatics are relevant prerequisites for systematic and effective qualification. Catalogue management needs to integrate different catalogues and support collaborative revisioning. The Health Informatics Learning Objectives Navigator (HI-LONa) offers an open, interoperable platform based on Semantic Web Technology. At present HI-LONa contains 983 learning objectives of three relevant catalogues. HILONa successfully supported a multiprofessional consensus process.
\end{abstract}

Keywords. Semantic Wikis, Semantic triplets, Learning objectives, Curriculum development

\section{Introduction}

The inexorable and irreversible digitalization of medicine drives an ever-increasing demand for highly qualified biomedical and health informatics (BMHI) professionals. In addition, BMHI literacy becomes indispensable in the health professions in general. Consented catalogues of BMHI learning objectives (BMHI CLOs) enable and promote appropriate qualification. The IMIA catalogue is a relevant example [1]. BMHI CLOs need to define a stable ground for curriculum development, teachers' and learners' orientation, and program comparison/evaluation, but must also keep pace with advances in the field and changing demands. For this purpose, the HI-LONa-project supports an agile, collaborative revision and consensus process. The HI-LONa platform provides BMHI CLOs as open linkable data and consequently uses Semantic Web Technology.

\section{Methods}

HI-LONa manages BMHI CLOs using a semantic wiki, i.e. wiki content linked by a conceptual network of SPO ("subject-predicate/relation-object") statements. The

\footnotetext{
${ }^{1}$ Corresponding author, Cord Spreckelsen, Institute of Medical Statistics, Computer and Data Sciences, Jena University Hospital, Bachstraße 18, 07743 Jena, Germany; E-mail: cord.spreckelsen@med.uni-jena.de.
} 
platform supports collaboration and consensus processes through low-threshold collaborative editing of CLOs, version control and a detailed change log. Comment functions guide revision steps. Similar learning objectives can be linked, viewed and compared across catalogs (horizontal linking) and with predecessors (vertical linking). HI-LONa can serve as a SPARQL endpoint and, thus, provides open, standardized access. For quality assurance, HI-LONa combines the flexibility of a wiki system with formbased structured data entry. Dynamic views based on semantic queries support content checks, comparison, and orientation. Quality assurance and consensus building is fostered using HI-LONa in nominal group processes with stakeholders of the German Medical Informatics Initiative and the BMHI related national scientific society (GMDS).

\section{Results}

HI-LONa contains a total of 983 learning objectives of three BMHI CLOs: It integrates the IMIA-Recommendations (version 2010), NKLM-MI (versions 2015 \& 2020) and the SMITH-BMHI catalogue. The latter is the result of a broad consensus process organized by the SMITH consortium of the nationally funded German Medical Informatics Initiative. On European level a usability test has been started by describing the BMHI program in Montenegro with this tool. Learning objectives as well as entire catalogues can be referenced via deep links (individual URLs). Thus, they can be dynamically included in resources hosted elsewhere. HI-LONa was implemented using Semantic MediaWiki 3.1.6 and MediaWiki 1.31.1 on an Ubuntu 18.04.7 server.

\section{Discussion}

Several approaches - especially in the field of medical CLOs - support web-based access and curriculum mapping [2]. In contrast to HI-LONa, these IT-platforms lack dedicated means for supporting agile revision processes, for semantically integrating different versions and entire catalogues, and for providing standardized access to machine interpretable and human readable content.

\section{Conclusion}

HI-LONa integrates relevant existing catalogues and supports an agile, collaborative revision and consensus process. It offers an open, interoperable platform for joining, managing, and further developing BMHI CLOs to cover future professional demands.

\section{References}

[1] Mantas J, et al. Recommendations of the International Medical Informatics Association (IMIA) on Education in Biomedical and Health Informatics. First Revision. Methods Inf Med. 2010;49(2):105-20.

[2] Balzer F, et al. Development and alignment of undergraduate medical curricula in a web-based, dynamic Learning Opportunities, Objectives and Outcome Platform (LOOOP). Med Teach. 2016;38(4):369-77. 